\title{
Functional recovery after radical prostatectomy for prostate cancer
}

\author{
Young Hwii Ko \\ Department of Urology, Yeungnam University College of Medicine, Daegu, Korea
}

\begin{abstract}
With the enthusiasm regarding robotic application in radical prostatectomy in accordance with the widespread use of serum prostate-specific antigen as a screening test, the number of surgeries performed for complete removal of the gland is increasing continuously. However, owing to the adjacent anatomical location of the prostate to the nerve and urethral sphincter complex, functional recovery, namely improvement from post-prostatectomy incontinence (PPI) and post-prostatectomy erectile dysfunction, still remains a main problem for patients who are reluctant to undergo surgery and tend to choose alternative ways instead. Since the late 1980s, the introduction of radical prostatectomy by open surgical modalities, the depth of the anatomical understanding of the structure surrounding the prostate is getting tremendous, which leads to the development of new surgical modalities and techniques that are consequently aimed at reducing the incidences of PPI and erectile dysfunction. Briefly, recent data from robotic radical prostatectomy, particularly on PPI, are quite acceptable, but by contrast, the reported potency regain rate still remains $<20 \%$, which indicates the need for advanced surgical modification to overcome it. In this review, the authors summarized the recent findings on the anatomy and surgical techniques reported up to now.
\end{abstract}

Keywords: Erectile dysfunction; Post-prostatectomy incontinence; Prostate cancer; Radical prostatectomy; Robotic surgery

\section{INTRODUCTION}

Prostate cancer is a common representative male cancer; in 2013, it was reported to be the second most common cancer in males, accounting for approximately $15 \%$ of male cancers [1]. Especially in the United States, where most data on prostate cancer have been accumulated, 233,000 persons developed prostate cancer in 2014, of whom 29,480 are predicted to die of prostate cancer [2]. Owing to its high prevalence, awareness of prostate cancer is high in the Western society. Therefore, societal interest in prostate cancer is also

Received: April 16, 2018, Revised: June 12, 2018

Accepted: August 23, 2018

Corresponding Author: Young Hwii Ko, Department of Urology, Yeungnam University College of Medicine, 170, Hyeonchung-ro, Nam-gu, Daegu 42415, Korea

Tel: +82-53-620-3695, Fax: +82-53-627-5535

E-mail: urokyh@naver.com predicted to increase gradually in Korea, where the population is rapidly aging because of the increased life expectancy and lifestyle patterns are becoming increasingly westernized. Indeed, in a prevalence survey published by the Korean Urological Association in 2009, the age-adjusted detection rate for suspected prostate cancer in 10,363 males aged $\geq 55$ years was $3.17 \%$, which was higher than that in Japan or China. In particular, the size of the high-risk group was reported to be larger than those in reports from Western countries $[3,4]$.

National cancer registration statistics show that $>9,000$ patients developed prostate cancer annually from 2009 to 2014; this was the fifth most common male cancer overall and the second highest incidence, after thyroid cancer, when adjusting for age. The growing prevalence of prostate cancer and the relatively large number of high-risk patients have been accompanied by an increase in the use rate of radical prostatectomy as treatment. Alongside the recent improvements in the availability of robot-assisted radical prostatec-

Copyright (C) 2018 Yeungnam University College of Medicine

This is an Open Access article distributed under the terms of the Creative Commons Attribution Non-Commercial License (http://creativecommons.org/licenses/by-nc/4.0/) which permits unrestricted non-commercial use, distribution, and reproduction in any medium, provided the original work is properly cited. 
tomy (RARP) based on a noninvasive approach, this has led to an increasing number of patients in Korea who receive radical surgery. However, radical prostatectomy is inevitably associated with adverse functional effects postoperatively, such as incontinence and impaired sexual function. For both patients and surgeons, in addition to the oncological objectives, functional recovery is being recognized as an important goal of surgery. To this end, in the present literature review, we aimed to summarize the results of recent studies and systematic reviews regarding these two postoperative functional aims. Most recent reports have focused on RARP rather than the open approach; thus, unless otherwise stated, the papers analyzed in this review pertain to the postoperative outcomes of RARP.

\section{Post-prostatectomy incontinence (PPI)}

\section{Causes and frequency}

As the prostate gland consists of the distal part of the male urethra, it is adjacent to several anatomical structures involved in maintaining urinary continence. As a result, in cases of resection involving the surrounding area, which is the aim of radical prostatectomy, the more successful the process of resection, the greater the risk of impairing urinary continence due to unintended damage to surrounding structures. Considering the close involvement of periprostatic structures in urinary continence, in most patients, post-prostatectomy incontinence (PPI) shows a pattern of early occurrence followed by gradual recovery over time. Recovery from PPI is usually evaluated by asking the patient about incontinence pad usage, rather than obtaining objective evidence via a 24-hour pad test. This means that the reported incidence is affected by the duration of the investigation and the types of questions. To date, the incidence reported by different surgeons considerably varies, in the range of $8-77 \%$ [5], but this seems to be, in part, due to authors using different definitions. Specifically, up to the early 2000s, the most common definition of recovery from incontinence was using 0 or 1 pad per day postoperatively, and this typically included the use of "security" pads. This led to a large number of patients being classified as having recovered from incontinence; however, major differences in quality of life were observed between patients who still used pads in daily living and those who did not, so this classification was criticized for only making surgeons' outcomes appear more favorable. Indeed, one study found that among 1,616 patients who were followed up for a mean of 50.7 months (range, 12-216 months) after open retropubic prostatectomy, 1,459 (90.3\%) reported experiencing urinary leakage, which clearly shows the enormous effect of the definition of the prevalence of the condition [6. With regard to the appropriate definition of PPI, Ahlering et al. divided 500 patients who had undergone RARP into groups with daily pad usages of 0 and 1 , and reported a significant difference in quality of life between the groups (1.16 vs. $3.41, p<0.0005)$, arguing, on this basis, that recovery from PPI should be defined as using 0 pads per day [7]. Likewise, the definitions of survival, continence, and potency (SCP) proposed in 2012 by world-leading surgeons as objective indicators to evaluate the outcomes of radical prostatectomy also consider 0 pad usage to be the standard definition for recovery from PPI [8]. However, different authors are still using their own definitions in studies published since then; thus, some caution is required when interpreting individual studies.

\section{Anatomical mechanisms of male urinary continence}

Although we still cannot claim to fully understand the mechanisms of urinary continence in males, alongside advances in radical prostatectomy techniques and increased numbers of operations, a large amount of related anatomical knowledge has been accumulated. In the 1980s, on the basis of the classical anatomical theory, the urogenital diaphragm, lying flat below the prostate gland, was thought to be a key structure in urinary continence by acting as the urethral sphincter; however, at the end of the 1990s, human and cadaver studies showed that the structure previously identified as the urogenital diaphragm did not exist $[9,10]$.

In terms of our present understanding of the mechanisms of male urinary continence, a review published in European Urology in 2010 provides a representative collection of results, and this review was updated in 2016 with the latest research [11,12].

Currently, male urinary continence is understood to be achieved by the combined actions of multiple anatomical structures surrounding the prostate gland; below, we summarize the major constituent muscular structures in this urethral 
sphincter complex and their roles.

(1) The smooth muscle sphincter (lissosphincter) consists of two layers (inner longitudinal and outer circular) and is innervated by the autonomous nervous system. It forms a spongy structure below the urethral mucosa, and external contraction completely cuts off the flow of urine. (2) The stratified sphincter (rhabdosphincter; the posterior part forms the median fibrous raphe [MFR] with no muscle layer) is responsible for the slow-twitch, passive control. It forms a cylindrical shape that originates from the prostate apex and attaches to the deep transverse perineal muscles. In the transverse crosssection, the muscle in the stratified sphincter is distributed in an omega shape; posteriorly, there is no muscle, but instead, forming the MFR are dense fibrous tissues. The MFR forms a posterior support complex by connecting to the central tendon posteriorly and Denonvilliers' fascia superiorly. (3) The puboperinealis muscle is responsible for fast-twitch, active control. It forms the medial part of the levator ani muscle. In the coronary view of magnetic resonance imaging, the puboperinealis muscle appears as two teardrop shapes running bilaterally, lateral to the urethra. As it attaches to the perineal body posterior to the urethra, the puboperinealis muscle ultimately forms a structure that supports the urethra [13].

\section{Correcting PPI based on anatomical understanding: development of surgical techniques and clinical outcomes}

On the basis of the understanding of the urethral sphincter complex described earlier, widespread efforts have been made to mitigate PPI by identifying and preserving this complex during surgery. By reviewing the current literatures, the techniques available during radical prostatectomy can be identified [14]. Conceptually, these techniques can be summarized as preservation of the internal/external sphincters and reconstruction of anterior/posterior support structures.

\section{1) Preservation of the bladder neck}

The bladder neck is a structure that includes the intravesical sphincter; in open surgery, broad incision and dissection of the bladder neck is performed to allow complete dissection of the prostate base, followed by suture ligation to restore the original thickness. However, the proximal internal urethral sphincter, previously referred to as the internal sphincter, was named the vesical sphincter, as it was reported to play a role in urinary continence [12,15]. Many comparative studies have been published that investigated surgical methods that maintain the anatomical structure of the bladder neck without any artificial manipulation. In a 2012 study by Friedlander et al., which included the largest number of patients, among 1,067 patients who underwent RARP between 2005 and 2011, 791 who underwent bladder neck-sparing surgery showed a significantly shorter time to urinary continence than the 276 patients who underwent conventional surgery [16]. In a systematic review of 13 studies on this topic, 1,130 patients in whom the bladder neck was spared were compared with 1,154 control patients and showed a significant improvement in the rate of urinary continence at both 6 and 12 months of follow-up [17]. However, bladder neck sparing is not possible in all the patients, and in patients who have previously undergone endoscopic prostate surgery such as transurethral resection of the prostate or in patients with a medial lobe, proper reconstruction of the bladder neck after resection is essential.

\section{2) Preservation of the neurovascular bundle}

From the perspective of classical anatomy, preservation of the neurovascular bundle (NVB) is intended to facilitate early recovery of erectile function rather than to prevent incontinence. However, preservation of the NVB is consistently reported to help recovery from PPI. Ko et al. analyzed 1,299 patients operated on by a single surgeon using the same technique and found that recovery from PPI, defined as 0 pad usage within 3 months, was significantly earlier in patients for whom the NVB was even partially spared than in patients with no NVB sparing [18]. A recent systematic review also demonstrated the association between NVB sparing and recovery from PPI [19]; thus, unless NVB sparing is impossible oncologically owing to the presence of aggressive cancer, preservation of the NVB even partially is important to aid recovery from PPI.

\section{3) Meticulous apical dissection}

The prostate apex, which is connected to the urethra, communicates immediately with the smooth muscle sphincter and 
stratified sphincter, so partial dissection of the prostate while sparing these structures is directly related to functional recovery.

\section{4) Sparing of the external sphincter}

As explained in the discussion of the anatomical structure, the puboperinealis muscle forms a hammock structure that supports the urethra as one aspect of urinary continence. Thus, preservation of the puboperinealis muscle could provide functional benefits in the prevention of PPI. Attached to the lateral part of the prostate apex, this muscle layer should be dissected under careful observation. Takenaka et al. reported that sparing the puboperinealis muscle could reduce the time to recovery from PPI [20].

\section{5) Posterior reconstruction and preservation of supporting structures}

As anatomical knowledge about the prostate gland increased, the importance of the posterior supporting structures, particularly the posterior support complex, has received greater attention. Posterior reconstruction has become a major milestone in modern prostate surgery; this procedure is also called the Rocco stitch, named after the surgeon who first introduced the concept of reconstructing the posterior structures that are damaged or lost while operating on the prostate [21]. Patel et al. extended this concept to include anterior reconstruction, as the role of the puboprostatic ligament, superior to the prostate apex, is also important in supporting the urethra [22]. Recently, the usefulness of the so-called total anatomical reconstruction has been reported, in which the prostate apex is divided into 5 anatomical units, each of which is reconstructed [23].

\section{6) Maximal preservation of the urethral length}

Ahlering et al. highlighted the remaining length of the urethra after resection of the prostate apex as an important factor in urinary continence [24]. Maintaining urethral length during surgery is essential to facilitate perfect urethrovesical anastomosis, which is one of the most important and demanding aspects of prostatectomy in terms of technical skill. In the dissection of the prostate apex, the final stage of prosta- tectomy, thorough dissection of the urethra before completely isolating the prostate is crucial to ensure enough urethral length. Meanwhile, unlike other parts of the prostate, the apex is not covered by the prostate capsule, so when cancer develops in this area, the risk of residual cancer after surgery is high. Preserving the length of the urethra to spare function can sometimes increase the risk of residual cancer, so when prostate cancer is confirmed to be located in the apex by using histological tests or preoperative radiography, cutting the apex as close to the urethra as possible is recommended.

\section{7) Secure urethrovesical anastomosis}

The greatest change and development with the introduction of RARP was, first, that a safe and complete urethrovesical anastomosis became possible as compared with the previous methods. In the age of open surgery, anastomosis was performed using interrupted stitches in 6 places, but now, by using a continuous stitch, the incidence of leakage has been reduced considerably. This change has led to a decrease in the incidence rates of urethral stricture and bladder neck contracture, which were previously common in the era of open retropubic prostatectomy.

\section{8) Regenerative materials}

In addition to surgical techniques, another strategy that can be expected to ameliorate PPI is to minimize surgical trauma and to maximize the healing process. Active research has been conducted on techniques such as stem cell injection, but the most successful clinical effort to date was the use of dehydrated human amnion/chorion membrane allograft by Patel et al., reported in 2015. In PPI, defined by the use of safety pads, groups that received the intervention for 1.2 and 1.8 months showed significantly shorter recovery times from incontinence [25], and in a subsequent study on 235 more patients, the use of this material during surgery was reported to have a similar effect of shortening the time to recovery of urinary continence and erectile function [26.

\section{9) Rehabilitative approaches: emphasis on Kegel exercises}

Although the most important factor is to thoroughly apply 
the aforementioned techniques during surgery and perform the techniques at a high level, exercise can be considered as another representative factor that can affect early postoperative recovery from PPI, and Kegel exercises, which can strengthen the urethral sphincter complex, are especially important. In 66 patients who had undergone open retropubic prostatectomy, Park et al. reported statistically significant differences in the extent of PPI, measured by a 24-hour pad test, between a control group and patients who had performed Kegel exercises for 1 hour once a week for 12 weeks [27]. As Kegel exercises can promote considerable recovery from incontinence even when performed sometime after surgery, postoperative patient education and follow-up of exercise levels should not be overlooked in outpatient care after radical prostatectomy.

\section{Postprostatectomy erectile dysfunction}

\section{Frequency and limitations of assessment}

Considering that problems with incontinence can have a major effect on quality of life, functional recovery is a primary objective. The 1-year recovery rate is reported to be $>90 \%$ due to surgical measures based on the improved anatomical knowledge discussed earlier, and of those patients who were still showing incontinence after the first postoperative year, approximately half recover their PPI within 3 years postoperatively [28]. Conversely, the recovery rates from postoperative erectile dysfunction reported in several papers do not exceed 10-29\%. Although several causes have been identified, the low recovery rate from erectile dysfunction is, unlike incontinence, largely due to the lack of consistent criteria for recovery. Approximately half of the reports on this topic up to 2009 used self-reports of successful sexual relations by the patients as evaluation criteria for recovery; $37 \%$ of the reports did not even indicate the criteria for potency recovery [29], and those that did state the criteria for recovery used very different criteria. Even among studies that used the International Index of Erectile Function (IIEF) 5, which is the most commonly used questionnaire for erectile function, different cutoff scores of 21, 17, and 15 were used. Moreover, many researchers, whether using the established IIEF or a less established questionnaire, only select some questions to use in the assessment. Among the various criteria proposed to date, erection sufficient for intercourse (ESI) is one of the most com- monly used definitions and has been used most commonly in reports on robotic surgery; however, objectivity is difficult to achieve because this is not a quantifiable index.

In recent reports, the second leading cause of the low recovery rate from erectile dysfunction is that unlike urinary incontinence, preoperative function cannot easily be assessed postoperatively in all patients. In other words, age, preoperative function measured with the IIEF-5, and the extent of intraoperative NVB sparing are well-proven independent factors that directly affect recovery of erectile function. However, because these variables differ among individuals, consistent postoperative improvements are difficult to identify. Thus, to control for these variables, most reports on erectile dysfunction have only included a subset of patients such as those aged $<60$ years; those with normal preoperative erectile function, defined as an IEF-5 score of at least 22; and those with complete bilateral preservation of the NVB during surgery. However, the patients satisfying these conditions typically only account for $<10 \%$ of all patients [30].

The third factor that affects the recovery rate from erectile dysfunction is the use of phosphodiesterase (PDE)-5 inhibitors. Although it is true that the release of sildenafil in 1997 revolutionized the treatment of erectile dysfunction, various types of PDE-5 inhibitor are available, and as they have become established as the primary treatment for erectile dysfunction, postoperative improvements tended to be considered in erectile function as recovery irrespective of whether or not the patient is taking PDE-5 inhibitors. Salonia et al. criticized this trend while arguing that the effects of PDE-5 inhibitors and spontaneous recovery of erectile function should be differentiated [31].

To resolve the issues discussed earlier, in 2012, authorities in the field of prostate surgery proposed the SCP system, which can consistently describe functional and oncological outcomes after radical prostatectomy. In this system, $S$ is classified as $\mathrm{S} 1$ if the prostate-specific antigen (PSA) level is $\geq 0.2$ $\mathrm{ng} / \mathrm{mL}$; C is classified as $\mathrm{C} 0$ in cases of 0 pad usage, as mentioned in the discussion of incontinence; and $\mathrm{P}$ is classified as P0 for scores of $\geq 18$ in the Sexual Health Inventory for Men (SHIM) without medication, and P1 for SHIM scores of $\geq 18$ while using a PDE-5 inhibitor [8]. This raised expectations that it would be possible, by limiting subjective assessment factors that depend on the researcher, to quantitatively evaluate erectile function, but still only few reports used this 
system. Nevertheless, the system is important insofar as it provides criteria for erectile function that can be perceived objectively in the future, and cutoff values based on SHIM, in particular, provide a direction for future studies in the field.

\section{Causes and surgical considerations for prevention}

Although the development of urinary incontinence can be explained by intraoperative injury to various structures affecting urinary continence, postprostatectomy erectile dysfunction has more simple causes, and the most important factor is injury to the cavernosal nerves. These nerves are the most important structures in maintaining healthy erectile function; owing to their anatomical relationship with the prostate, running in the 3 and 9 o'clock directions from the apex of the prostate, and along the posterolateral aspect of the prostate body, injury during radical prostatectomy is inevitable, and this results in postoperative erectile dysfunction. Mechanisms for this nerve injury include unintended physical damage to the nerve itself during surgery, but the extent of nerve injury is reported to differ, and neuropraxia, in particular, is known as a representative mechanism of nerve injury capable of recovery and rehabilitation [32]. Owing to continual efforts to minimize intraoperative nerve injury, when complete resection is not oncologically necessary in patients with preserved preoperative erectile function, bilateral nerve-sparing surgery has become a major trend in modern surgery. Minimizing unintended intraoperative damage to the $\mathrm{NBV}$ is considered the most important principle in preserving erectile function.

Meanwhile, fibrotic remodeling, cavernous smooth muscle apoptosis, and ischemic nerve injury can also have negative effects on the recovery of erectile function, and the use of various regenerative materials has recently been reported as another potential strategy to restrict the inflammatory response and promote regeneration.

\section{Detailed postoperative treatment strategies}

\section{1) Use of PDE-5 inhibitors}

As mentioned earlier, as PDE-5 inhibitors have become a commonplace treatment strategy in patients with erectile dys- function, given that some degree of nerve injury and erectile dysfunction is inevitable during surgery, penile rehabilitation strategies have been proposed by using these drugs at the earliest possible stage for rehabilitative purposes [31]. Nevertheless, the ultimate goal of penile rehabilitation is not continual use of PDE-5 inhibitors but spontaneous recovery of erectile function without depending on medications, and research has begun to focus on this. Up to now, many studies have been conducted on on-demand therapies using different drugs and daily dosing methods [33-43], but most of the results have been negative. Thus, although medication has shown amelioration of symptoms irrespective of the drug type or method of administration, most randomized clinical trials have reported a decline in erectile function after a 2-month drug holiday. Therefore, unlike initial expectations, recovery of erectile function using PDE-5 inhibitors is not currently considered successful. On the other hand, most of the studies used a short medication period of $<1$ year and, given that the typical time until postoperative recovery of erectile function is around 2 years, it is still difficult to make conclusions about the long-term effects of these drugs [44]. In 2015, the International Consultation for Sexual Medicine reviewed randomized trials on this topic, and their recommendations also did not reach a conclusion about whether the PDE-5 inhibitors help recover erectile function and, similarly, stated that the data obtained were inadequate to determine the formulations that should be used in penile rehabilitation [31].

\section{2) Strategies other than PDE-5 inhibitors}

Although the convenience of oral erectile dysfunction treatments is difficult to replace, formulations that have other pharmacological or pharmacokinetic mechanisms can be attempted in patients who show a poor response to oral formulations. Possible alternatives to oral drugs include intracavernosal injection (ICI), transurethral alprostadil, use of a vacuum erectile device (VED), male hormone replacement therapy, and stem cell therapy. However, apart from ICI and VED, these treatments can only be used in very restricted cases in Korea. The use of ICI in postoperative erectile dysfunction was first reported by Montersi et al. in 1997 [45]; since then, significant erectile function recovery has been reported by other researchers, but the studies have been relatively smaller 
than those that used PDE-5 inhibitors, with recent reports of non-significant results [46]. Thus, further research will be required for the clinical application of ICI.

Although rarely used for clinical purposes in Korea, VED is noninvasive and can be effective regardless of the mechanism of erectile dysfunction. Studies on VED use after prostate surgery are few, but the researchers who have investigated VED all reported increases in penile length $[47,48]$.

\section{CONCLUSION}

Owing to the increased incidence of prostate cancer due to aging, heightened social interest, and the increase in individuals at high risk of cancer that was recently reported in Korea, radical prostatectomy is expected to become increasingly common. As this procedure is performed more in elderly patients, postoperative functional recovery will become a more important task in clinical care. As we have seen in this review, the incidence of postoperative incontinence has been reduced greatly by focusing on the development of surgical techniques based on anatomical knowledge and advancements in technology. The duration of disease has also decreased considerably since the introduction of robotic surgery.

By contrast, erectile dysfunction remains a task to be resolved in a large number of patients who require prostatectomy. In spite of great expectations, PDE-5 inhibitors, which have been the focus of attention as an oral treatment for erectile dysfunction, have failed to show clear results, and much research is still required on dosing methods and treatment duration. In addition to erectile dysfunction, many patients experience postoperative complications in other aspects of their sex lives, with over half of patients experiencing changes in the sensation of orgasm, decreased sexual appetite, increased penis size, Peyronie's disease, or climacturia, indicating that this will remain a challenging problem for some time [31]. Going forward, we will need to conduct further research on postoperative functional changes, focusing not only on erectile dysfunction but also on sexual quality of life.

\section{CONFLICT OF INTEREST}

No potential conflict of interest relevant to this article was reported.

\section{ORCID}

Young Hwii Ko, https://orcid.org/0000-0002-9150-4292

\section{REFERENCES}

1. Siegel R, Ma J, Zou Z, Jemal A. Cancer statistics, 2014. CA Cancer J Clin 2014;64:9-29.

2. Ferlay J, Soerjomataram I, Dikshit R, Eser S, Mathers C, Rebelo M, et al. Cancer incidence and mortality worldwide: sources, methods and major patterns in GLOBOCAN 2012. Int J Cancer 2015;136:E359-86.

3. Song C, Kang T, Lee MS, Ro JY, Lee SE, Lee E, et al. Clinicopathological characteristics of prostate cancer in Korean men and nomograms for the prediction of the pathological stage of the clinically localized prostate cancer: a multi-institutional update. Korean J Urol 2007;48:125-30. Korean.

4. Song K, Song C, Ahn H. Continuing trends of the clinical parameter migration in patients with prostate cancer in Korea. Korean J Urol 2007;48:574-8. Korean.

5. Klingler HC, Marberger M. Incontinence after radical prostatectomy: surgical treatment options. Curr Opin Urol 2006; 16:60-4.

6. Peterson AC, Chen Y. Patient reported incontinence after radical prostatectomy is more common than expected and not associated with the nerve sparing technique: results from the Center for Prostate Disease Research (CPDR) database. Neurourol Urodyn 2012;31:60-3.

7. Liss MA, Osann K, Canvasser N, Chu W, Chang A, Gan J, et al. Continence definition after radical prostatectomy using urinary quality of life: evaluation of patient reported validated questionnaires. J Urol 2010;183:1464-8.

8. Ficarra V, Sooriakumaran P, Novara G, Schatloff O, Briganti A, Van der Poel H, et al. Systematic review of methods for reporting combined outcomes after radical prostatectomy and proposal of a novel system: the survival, continence, and potency (SCP) classification. Eur Urol 2012;61:541-8.

9. Kaye KW, Milne N, Creed K, van der Werf B. The 'urogenital diaphragm', external urethral sphincter and radical prostatectomy. Aust N Z J Surg 1997;67:40-4.

10. Dorschner W, Biesold M, Schmidt F, Stolzenburg JU. The dispute about the external sphincter and the urogenital diaphragm. J Urol 1999;162:1942-5.

11. Walz J, Burnett AL, Costello AJ, Eastham JA, Graefen M, Guillonneau B, et al. A critical analysis of the current knowledge of surgical anatomy related to optimization of cancer control and preservation of continence and erection in candidates for radical prostatectomy. Eur Urol 2010;57:179-92.

12. Walz J, Epstein JI, Ganzer R, Graefen M, Guazzoni G, Kaouk $\mathrm{J}$, et al. A critical analysis of the current knowledge of surgical anatomy of the prostate related to optimisation of cancer control and preservation of continence and erection in candidates for radical prostatectomy: an update. Eur Urol 2016;70:301-11. 13. Myers RP, Cahill DR, Kay PA, Camp JJ, Devine RM, King 
BF, et al. Puboperineales: muscular boundaries of the male urogenital hiatus in 3D from magnetic resonance imaging. J Urol 2000;164:1412-5.

14. Sridhar AN, Abozaid M, Rajan P, Sooriakumaran P, Shaw G, Nathan S, et al. Surgical techniques to optimize early urinary continence recovery post robot assisted radical prostatectomy for prostate cancer. Curr Urol Rep 2017;18:71.

15. Dorschner W, Stolzenburg JU, Neuhaus J. Structure and function of the bladder neck. Adv Anat Embryol Cell Biol 2001; 159:III-XII, 1-109.

16. Friedlander DF, Alemozaffar M, Hevelone ND, Lipsitz SR, $\mathrm{Hu}$ JC. Stepwise description and outcomes of bladder neck sparing during robot-assisted laparoscopic radical prostatectomy. J Urol 2012;188:1754-60.

17. Ma X, Tang K, Yang C, Wu G, Xu N, Wang M, et al. Bladder neck preservation improves time to continence after radical prostatectomy: a systematic review and meta-analysis. Oncotarget 2016;7:67463-75.

18. Ko YH, Coelho RF, Chauhan S, Sivaraman A, Schatloff O, Cheon J, et al. Factors affecting return of continence 3 months after robot-assisted radical prostatectomy: analysis from a large, prospective data by a single surgeon. J Urol 2012;187: 190-4.

19. Reeves F, Preece P, Kapoor J, Everaerts W, Murphy DG, Corcoran NM, et al. Preservation of the neurovascular bundles is associated with improved time to continence after radical prostatectomy but not long-term continence rates: results of a systematic review and meta-analysis. Eur Urol 2015;68: 692-704.

20. Takenaka A, Tewari AK, Leung RA, Bigelow K, El-Tabey N, Murakami G, et al. Preservation of the puboprostatic collar and puboperineoplasty for early recovery of urinary continence after robotic prostatectomy: anatomic basis and preliminary outcomes. Eur Urol 2007;51:433-40.

21. Rocco F, Carmignani L, Acquati P, Gadda F, Dell'Orto P, Rocco B, et al. Early continence recovery after open radical prostatectomy with restoration of the posterior aspect of the rhabdosphincter. Eur Urol 2007;52:376-83.

22. Patel VR, Coelho RF, Palmer KJ, Rocco B. Periurethral suspension stitch during robot-assisted laparoscopic radical prostatectomy: description of the technique and continence outcomes. Eur Urol 2009;56:472-8.

23. Porpiglia F, Bertolo R, Manfredi M, De Luca S, Checcucci E, Morra I, et al. Total anatomical reconstruction during robot-assisted radical prostatectomy: implications on early recovery of urinary continence. Eur Urol 2016;69:485-95.

24. Borin JF, Skarecky DW, Narula N, Ahlering TE. Impact of urethral stump length on continence and positive surgical margins in robot-assisted laparoscopic prostatectomy. Urology 2007;70:173-7.

25. Patel VR, Samavedi S, Bates AS, Kumar A, Coelho R, Rocco $\mathrm{B}$, et al. Dehydrated human amnion/chorion membrane allograft nerve wrap around the prostatic neurovascular bundle accelerates early return to continence and potency following robot-assisted radical prostatectomy: propensity score-matched analysis. Eur Urol 2015;67:977-80.
26. Ogaya-Pinies G, Palayapalam-Ganapathi H, Rogers T, Hernandez-Cardona E, Rocco B, Coelho RF, et al. Can dehydrated human amnion/chorion membrane accelerate the return to potency after a nerve-sparing robotic-assisted radical prostatectomy? Propensity score-matched analysis. J Robot Surg 2018;12:235-43.

27. Park SW, Kim TN, Nam JK, Ha HK, Shin DG, Lee W, et al. Recovery of overall exercise ability, quality of life, and continence after 12-week combined exercise intervention in elderly patients who underwent radical prostatectomy: a randomized controlled study. Urology 2012;80:299-305.

28. Mandel P, Preisser F, Graefen M, Steuber T, Salomon G, Haese A, et al. High chance of late recovery of urinary and erectile function beyond 12 months after radical prostatectomy. Eur Urol 2017;71:848-50.

29. Mulhall JP. Defining and reporting erectile function outcomes after radical prostatectomy: challenges and misconceptions. J Urol 2009;181:462-71.

30. Sridhar AN, Cathcart PJ, Yap T, Hines J, Nathan S, Briggs $\mathrm{TP}$, et al. Recovery of baseline erectile function in men following radical prostatectomy for high-risk prostate cancer: a prospective analysis using validated measures. J Sex Med 2016;13:435-43.

31. Salonia A, Adaikan G, Buvat J, Carrier S, El-Meliegy A, Hatzimouratidis K, et al. Sexual rehabilitation after treatment for prostate cancer-part 2: recommendations from the fourth International Consultation for Sexual Medicine (ICSM 2015). J Sex Med 2017;14:297-315.

32. Ahlering TE, Skarecky DW. Preserving sexual function after robotic radical prostatectomy: avoiding thermal energy near nerves. BJU Int 2014;114:131-2.

33. Montorsi F, Brock G, Lee J, Shapiro J, Van Poppel H, Graefen $\mathrm{M}$, et al. Effect of nightly versus on-demand vardenafil on recovery of erectile function in men following bilateral nervesparing radical prostatectomy. Eur Urol 2008;54:924-31.

34. Bannowsky A, Schulze H, van der Horst C, Hautmann S, Jünemann KP. Recovery of erectile function after nerve-sparing radical prostatectomy: improvement with nightly low-dose sildenafil. BJU Int 2008;101:1279-83.

35. Padma-Nathan H, McCullough AR, Levine LA, Lipshultz LI, Siegel R, Montorsi F, et al. Randomized, double-blind, placebo-controlled study of postoperative nightly sildenafil citrate for the prevention of erectile dysfunction after bilateral nervesparing radical prostatectomy. Int J Impot Res 2008;20:47986.

36. Pace G, Del Rosso A, Vicentini C. Penile rehabilitation therapy following radical prostatectomy. Disabil Rehabil 2010;32: 1204-8.

37. Aydogdu O, Gokce MI, Burgu B, Baltac S, Yaman O. Tadalafil rehabilitation therapy preserves penile size after bilateral nerve sparing radical retropubic prostatectomy. Int Braz J Urol 2011;37:336-44.

38. Bannowsky A, van Ahlen H, Loch T. Increasing the dose of vardenafil on a daily basis does not improve erectile function after unilateral nerve-sparing radical prostatectomy. J Sex Med 2012;9:1448-53. 
39. Montorsi F, Brock G, Stolzenburg JU, Mulhall J, Moncada I, Patel HR, et al. Effects of tadalafil treatment on erectile function recovery following bilateral nerve-sparing radical prostatectomy: a randomised placebo-controlled study (REACTT). Eur Urol 2014;65:587-96.

40. Nakano Y, Miyake H, Chiba K, Fujisawa M. Impact of penile rehabilitation with low-dose vardenafil on recovery of erectile function in Japanese men following nerve-sparing radical prostatectomy. Asian J Androl 2014;16:892-6.

41. Seo YE, Kim SD, Kim TH, Sung GT. The efficacy and safety of tadalafil $5 \mathrm{mg}$ once daily in the treatment of erectile dysfunction after robot-assisted laparoscopic radical prostatectomy: 1-year follow-up. Korean J Urol 2014;55:112-9.

42. Natali A, Masieri L, Lanciotti M, Giancane S, Vignolini G, Carini M, et al. A comparison of different oral therapies versus no treatment for erectile dysfunction in 196 radical nervesparing radical prostatectomy patients. Int J Impot Res 2015; 27:1-5.

43. Canat L, Güner B, Gürbüz C, Atış G, Çaşkurlu T. Effects of three-times-per-week versus on-demand tadalafil treatment on erectile function and continence recovery following bilateral nerve sparing radical prostatectomy: results of a prospective, randomized, and single-center study. Kaohsiung J
Med Sci 2015;31:90-5.

44. Liu C, Lopez DS, Chen M, Wang R. Penile rehabilitation therapy following radical prostatectomy: a meta-analysis. J Sex Med 2017;14:1496-503.

45. Montorsi F, Guazzoni G, Strambi LF, Da Pozzo LF, Nava L, Barbieri L, et al. Recovery of spontaneous erectile function after nerve-sparing radical retropubic prostatectomy with and without early intracavernous injections of alprostadil: results of a prospective, randomized trial. J Urol 1997;158:1408-10.

46. Mulhall J, Land S, Parker M, Waters WB, Flanigan RC. The use of an erectogenic pharmacotherapy regimen following radical prostatectomy improves recovery of spontaneous erectile function. J Sex Med 2005;2:532-40.

47. Raina R, Agarwal A, Ausmundson S, Lakin M, Nandipati KC, Montague DK, et al. Early use of vacuum constriction device following radical prostatectomy facilitates early sexual activity and potentially earlier return of erectile function. Int J Impot Res 2006;18:77-81.

48. Köhler TS, Pedro R, Hendlin K, Utz W, Ugarte R, Reddy P, et al. A pilot study on the early use of the vacuum erection device after radical retropubic prostatectomy. BJU Int 2007; 100:858-62. 